\title{
El fondo Aracy Abreu Amaral y la historiografía del arte de América Latina
}

[ The Aracy Abreu Amaral documentary fund and the historiography of Latin American art

\section{Renata Ribeiro dos Santos ${ }^{\mathrm{I}}$}

RESUMO - Este texto apresenta uma revisão inicial da pesquisa realizada no Fundo Aracy Abreu Amaral, sob custódia do Instituto de Estudos Brasileiros da Universidade de São Paulo. O objetivo fundamental do estudo é estabelecer relações e trocas intelectuais entre os diferentes agentes que, juntamente com Amaral, foram decisivos para a delimitação e difusão da arte latino-americana entre os anos I970 e I990. • PALAVRAS-CHAVE • Aracy Abreu Amaral; arte latino-americana; fundo documental; agente cultural. - ABSTRACT .
The present text presents an initial review of the research carried out at the Aracy Abreu Amaral Fund, guarded by the Instituto de Estudos Brasileiros of the University of São Paulo. The fundamental objective of the study is to establish the relations and intellectual exchanges between the different agents who, together with Amaral, were decisive in the delimitation and diffusion of Latin American art between the I970s and I990s. $\cdot$ KEYWORDS - Aracy Abreu Amaral; Latin American art; documentary fund; cultural agent.

Recebido em 20 de fevereiro de 2020

Aprovado em 6 de março de 2020

RIBEIRO DOS SANTOS, Renata. El fondo Aracy Abreu Amaral y la historiografía del arte de América Latina. Revista do Instituto de Estudos Brasileiros, Brasil, n. 75, p. 200-2Io, abr. 2020.

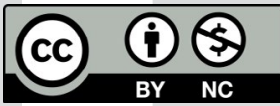

DOI: http://dx.doi.org/Io.II6o6/issn.23I6-90IX.voi75p200-2Io

I Universidade de Oviedo (UNIOVI, Oviedo, Principado de Astúrias, Espanha). 
El historiador de arte mexicano Xavier Moyssén² escribía en I978:

Es impresionante, y por demás significativo, el crecido número de mujeres que actualmente se dedican en Latinoamérica al ejercicio de la historia y la crítica de arte. Desde mi particular opinión, a la cabeza de tan respetables señoras se encuentra, en relación con el arte moderno, la brasileña Aracy Amaral, autora de una obra sólidamente establecida, tanto por su sabiduría como por lo ponderado y objetivo de sus juicios. (MOYSSÉN, I978, p. I85).

En línea con el argumento de Moyssén y, sin demasiado riesgo en equivocarse, se podría afirmar que la paulistana Aracy Abreu Amaral (São Paulo, I930) juntamente con otros importantes nombres de la historia, crítica y teoría del arte - como por ejemplo el argentino Damián Bayón, el peruano Juan Acha, la argentino-colombiana Marta Traba o la franco-chilena Nelly Richard - sentaron las pautas para el proceso de elaboración, sistematización, reconocimiento y escritura de una historiografía del arte de América Latina que, al día de hoy se entiende como una categoría consolidada.

La hipótesis de la existencia de un cuerpo de pensadores que, en un momento determinado desde América Latina, trataron de (re)elaborar su historia, no es novedosa e incluso podríamos decir que, entre los círculos latinoamericanistas, es bastante reconocida y aceptada. Entre tanto, al investigar el Fundo Aracy Abreu Amaral (AAA) custodiado por el Arquivo do Instituto de Estudos Brasileiros de la Universidade de São Paulo (IEB/USP), vemos materializado un real y prolijo diálogo entre estos pensadores. Lo que constituye un elemento fundamental para confirmar la teoría de una labor conjunta de elaboración historiográfica.

En este archivo personal se encuentran cerca de I5.500 documentos, fuentes referentes a su labor docente, curatorial, relaciones personales e investigación,

2 Xavier Moyssén (Morelia, I924 - Ciudad de México, 200I) fue un renombrado investigador y docente del Instituto de Investigaciones Estéticas de la Universidad Autónoma de México. Sus estudios se centraron sobretodo en el análisis del arte producido en México, desde su época colonial hasta las expresiones vanguardistas de la primera mitad del s. XX. El fragmento del texto citado es el primer párrafo de la reseña que hace Moyssén al libro Artes na Semana de 22, escrito por la Profa. Aracy Amaral en I970 y reeditado en 1976. 
entre muchas otras. En un primer contacto con este acervo es posible entender que, en Aracy Amaral, se conjugan una mentalidad investigadora y el completo entendimiento de lo que conlleva esta profesión. El conjunto documental presenta un arduo trabajo de sistematización, recopilación y conservación de los más distintos materiales que pasaron por su mano a lo largo de los años.

Este rigor metodológico y documental también lo observó Xavier Moyssén (I978, p. I86) que, en su ya citado texto, comentaba sobre el libro de Amaral que "resulta excepcional por el esmero y la pasión que puso a lo largo de la investigación, a fin de recoger los materiales tanto gráficos como informativos", listando a seguir las más diferentes fuentes utilizadas por la investigadora: desde viejas fotografías, caricaturas y dibujos de publicidad, hasta la realización de entrevistas a los protagonistas del periodo que estudiaba.

Realmente, igual variedad de fuentes se aprecia en el fondo AAA y, analizando en profundidad este gran corpus documental, es posible encontrar las claves para entender como algunos nombres trabajaron como agentes, defensores, activistas y fundadores creadores que, interrelacionados, fomentaron la elaboración de un relato sistematizado y establecido de lo que es (y se definió por) arte latinoamericano. En un previo y breve análisis de este conjunto documental podemos empezar a trazar los puentes y caminos de intercambio que se establecieron entre estos agentes.

Comencemos este deambular por Damián Bayón (Buenos Aires, I9I5 - Paris, I995), uno de los propulsores de los estudios relacionados al arte latinoamericano y, juntamente con Marta Traba - ambos discípulos de Jorge Romero Brest-, redactores (redactor en jefe en el caso de Bayón) de la revista Ver y Estimar, publicada entre 1949 y 1955. La propia Aracy Amaral fue quien comentó la importancia e influencia de aquella publicación, citando que "influyó en la formación de toda una generación de críticos y artistas no solo en la Argentina sino también en Uruguay (y en Brasil podría añadir) puesto que, en sus comienzos, en los años 50, yo misma coleccionaba y leía celosamente esta publicación, tan avanzada para la época" (AMARAL, I984, p. I7).

Dentro las muchas tareas llevadas a cabo por Bayón, sobresale la coordinación de un estudio encargado por la UNESCO sobre las artes plásticas en América Latina. Ideado dentro de la serie América Latina en su Cultura, se planteó una reunión de especialistas en las artes que pudiesen organizar una publicación que tratase de la producción estética de la región, siguiendo los pasos de las dos ediciones anteriores, referentes a la arquitectura y a la literatura. 


\section{América Latina en sus artes}

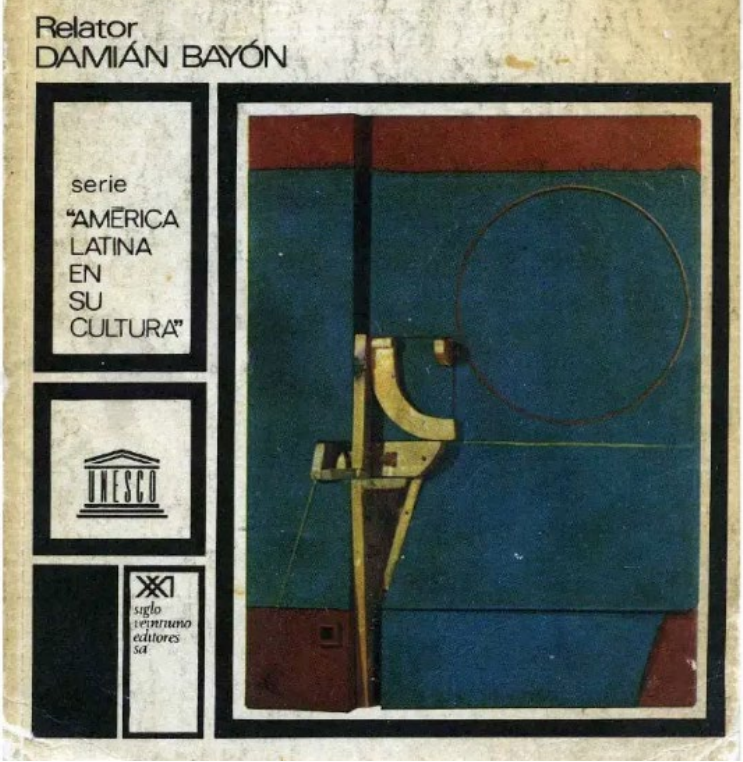

Figura I - Cubierta del libro América Latina en sus artes, coordinado por Damián Bayón y publicado en 1974 dentro de la serie América Latina en su Cultura, encargada por la Unesco

Finalmente, la reunión se realizó en Quito (22 a 26 de junio de I970) y tuvo como relator a Damián Bayón. De acuerdo con Aracy Amaral (I995), aquel fue el primer encuentro en congregar a críticos de arte de los distintos países latinoamericanos. Entre los expertos reunidos "a título personal, y no como representantes de gobiernos o instituciones" 3 figuran nombres como los de los brasileños Mario Barata y Mario Pedrosa, la cubana Adelaida de Juan y el mexicano Jorge Alberto Manrique ${ }^{4}$, como

3 Fundo Aracy Abreu Amaral (AAA), Arquivo do Instituto de Estudos Brasileiros de la Universidade de São Paulo (IEB/USP), código de referencia AAA-AL-ALSA-ooI.

4 La lista completa de los expertos que acudieron a la reunión es la siguiente: Jorge Enrique Adoum, Mario Barata, Damián Bayón, Víctor Carvacho, Edmundo Desnoes, Adelaida de Juan, Jorge Alberto Manrique, José Luis Martínez, Samuel Oliver, Mario Pedrosa, Carlos Rodríguez Saavedra y Filoteo Sarmiento. En la lista de participantes figuran también Observadores de la Asociación Internacional de Artes Plásticas y representantes del Secretariado y del Comité de la Delegación Ecuatoriana de la Unesco (AAA-AL-ALSA-ooI). 
podemos apreciar en el informe final de la Reunión del grupo de trabajo para el Estudio de las Artes Plásticas en América Latina5.

En aquella reunión, entre las diversas recomendaciones realizadas alrededor de la propuesta central - la elaboración del libro América Latina en sus artes (Figura I) -, se propuso nombres de expertos en arte de la región que deberían de encargarse de la redacción de los capítulos de la obra ${ }^{6}$. Por una carta posterior que le envió Bayón a Amaral, conocemos que la inclusión de su nombre para la redacción fue una propuesta directa de Mario Pedrosa7.

Para las cuestiones que tratamos en esta reflexión, resulta sugerente el cruce de los nombres propuestos para la redacción con el Anexo II ${ }^{8}$ del citado informe, donde se recoge, a manera de índice tentativo, los epígrafes que debería tener América Latina en sus artes. Estos datos ayudan a sentar las pautas de cómo, en los comienzos de años 70, se propone la sistematización, jerarquización y estudio del arte latinoamericano, presentado en un volumen único.

Finalmente, la publicación apareció en el año de I974 y en ella no figuraron muchos de estos nombres indicados para la redacción de los capítulos en aquella reunión inicial, como por ejemplo los de Aracy y Marta Traba (BAYÓN, I974).

En la correspondencia intercambiada entre Damián y Aracy, sabemos que, después de varias proposiciones de temas de trabajo a la brasileña y de la negación de un viaje por otros países de la región, Aracy Amaral declina la invitación inicial, incidiendo en el siguiente punto

[...] Al decir verdad, el tema es interesante, pero sin poder coger las informaciones directamente en los lugares necesarios no me parecería también posible, en tan poco tiempo, realizar algo bueno, efectivamente, sobre toda América Latina: como ud.

5 Fundo Aracy Abreu Amaral, Arquivo do IEB/USP, código de referencia AAA-AL-ALSA-001.

6 En el Informe Final figura el siguiente listada de posibles nombres para la redacción: Aracy Amaral, Miguel Arroyo, Alfredo Boulton, Luis Cardoza y Aragón, Leopoldo Castedo, Hernán Crespo, Jorge Juan Crespo de la Serna, Justino Fernández, Fermín Fevre, Juan García Ponce, Enrique Lihn, Tomás Maldonado, Federico Moraes, José Ricardo Morales Malva, Luis Oyarzún Peña, Julio Payró, Alberto Pérez, Clarival do Prado Valladares, Ida Rodríguez Prampolini, Antonio R. Romera, Jorge Romero Brest, Martha Traba (sic), Emilio Westphalen, Saúl Yurkievich, Walter Zanini (Fundo Aracy Abreu Amaral, Arquivo do IEB/USP, código de referencia AAA-AL-ALSA-OOI).

7 Fundo Aracy Abreu Amaral, Arquivo do IEB/USP, código de referencia AAA-AL-ALSA-003 - Carta de Damián Bayón a Aracy Amaral con fecha de 23/3/1972.

8 Fundo Aracy Abreu Amaral, Arquivo do IEB/USP, código de referencia AAA-AL-ALSA-0o2 - Anexo II - América Latina en sus artes, p. 7-Io. 
sabe, aunque semejante en muchos puntos, es bien distinta la situación en Uruguay, Argentina, Brasil y Venezuela, por ejemplo?.

Con esta aseveración, la historiadora deja patente que, si bien hubiera una voluntad de una elaboración de un cuerpo teórico conjunto sobre la producción estética de la región, este no podría estar supeditado a una voluntad homogeneizadora y sin matices locales.

Es posible hacer enlazar este proyecto de Bayón con Arquitectura neocolonial: América Latina, Caribe, Estados Unidos (Figura 2), coordinado por Aracy Amaral y editado por el Memorial da América Latina y el Fondo de Cultura Económica. Coincidentemente, el libro resultante de este proyecto fue publicado en I994, 20 años después de aquel ya canónico texto, coordinado por Bayón.

Dentro de los fondos AAA se encuentran más de 600 elementos relacionados a la concepción y consecución de ese libro ${ }^{\mathrm{IO}}$. Entre la rica y vasta correspondencia, nos deparamos con nombres fundamentales en el estudio de la arquitectura de América Latina, como puede ser el de Ramón Gutiérrez, Enrique Xavier de Anda Alanís, Roberto Segre, Alberto Petrina, Margarita Gutman, Germán Téllez, Jorge Alberto Manrique, Rita Eder, entre otros. La interrelación entre estos nombres evidencia dos supuestos: la reunión de los mayores (y más críticos) arquitectos e historiadores de la arquitectura para pensar la creación y los usos de lo neocolonial en América Latina; pero también la elaboración de textos que evidenciaban las características propias de cada territorio - sus particularidades - en diálogo con el conjunto de países de la región. Esto apunta a cierto carácter emancipador y no homogeneizante, - glocal dirían otros - que los Estudios Latinoamericanos caminarán entrada la década de los 90.

9 Fundo Aracy Abreu Amaral, Arquivo do IEB/USP, código de referencia AAA-AL-ALSA-044 - Carta de Aracy Amaral a Damián Bayón con fecha de I5/9/1972. 


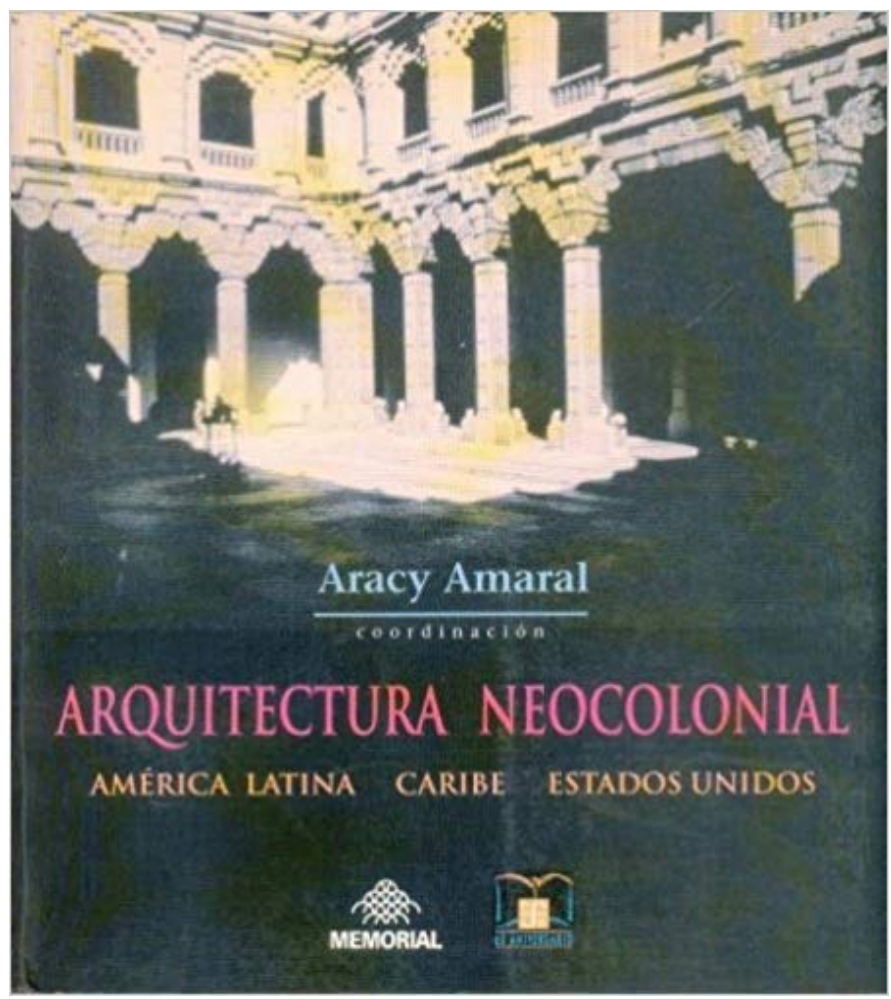

Figura 2 - Cubierta del libro Arquitectura neocolonial: América Latina, Caribe, Estados Unidos, coordinado por Aracy Amaral, publicado en 1994

Regresando a los años 70, momento de forja de lo "latinoamericano estético", tenemos como hito fundamental de este proceso la I Bienal Latino-americana de São Paulo (Figura 3), celebrada en $1978^{\text {II }}$. Prácticamente todos los nombres de intelectuales, críticos e historiadores citados en los párrafos anteriores estuvieron relacionados, directa o indirectamente, con la realización de este certamen o, en otros casos, se vieron implicados en la cancelación de su continuidad. El acervo recopilado por Amaral sobre este importantísimo y, por veces, olvidado evento reúne más de Ioo documentos - correspondencia, actas e informes de reuniones, artículos publicados en la prensa de época, que pueden ayudar a entender los ideales buscados en la elaboración de aquella bienal regional, a través del entramado de lazos y relaciones analizados en estas comunicaciones.

II La Bienal fue celebrada entre los días 3 de noviembre y I7 de diciembre de 1978 en el Pavilhão Armando de Arruda Pereira del Parque do Ibirapuera. Reunió a más de I60 artistas procedentes de I3 países: Argentina, Bolivia, Brasil, Chile, Colombia, El Salvador, Ecuador, Honduras, México, Paraguay, Perú, República Dominicana, Uruguay (FUNDAÇÃO BIENAL DE SÃO PAULO, I978). 


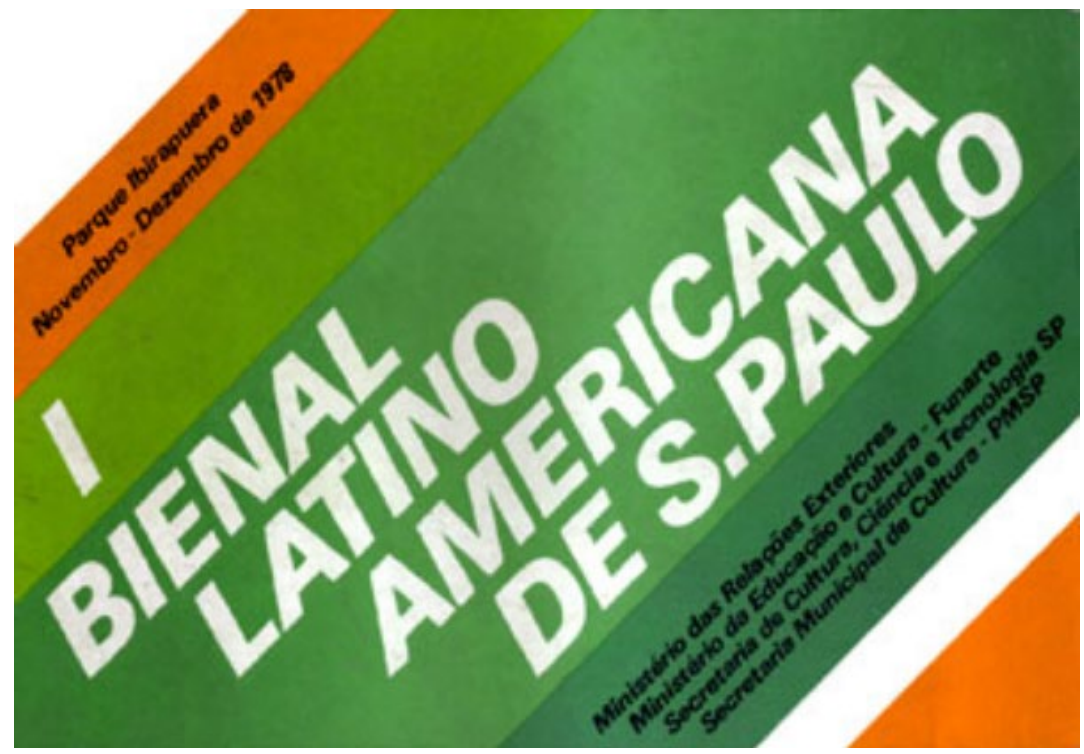

Figura 3-Cubierta del catálogo de la I Bienal Latinoamericana de São Paulo celebrada en el Parque do Ibirapuera en I978

La documentación nos ayuda a trazar la línea de actuación de estos agentes vinculados con la defensa y con la puesta en valor de un arte latinoamericano -, desde las pautas y sugerencias indicadas en las reuniones previas ocurridas en el año $\mathrm{I} 977^{\mathrm{I2}}$, pasando por las críticas e inclinaciones de las opiniones durante el evento ${ }^{\mathrm{I3}} \mathrm{y}$, finalmente, la cancelación de la siguiente edición en I980 y el lanzamiento de una convocatoria - impulsada en fuerte medida por Aracy Amaral - de la Reunião de Consulta de Críticos de Arte de América Latina ${ }^{\mathrm{I} 4}$ en el mismo año 80 en la ciudad paulistana.

Los críticos e intelectuales ahí reunidos - la creme de la creme de la crítica de arte latinoamericana - ${ }^{\mathrm{I}}$ tenían como punto clave de discusión el de deliberar sobre el devenir de la Bienal y cambiar o no su rumbo hacia una vitrina para el

I2 La primera reunión fue celebrada el II de septiembre de I977 con la participación de Aracy Amaral, Damián Bayón y Fábio Magalhães (Fundo Aracy Abreu Amaral, Arquivo do IEB/USP, código de referencia AAA-BIELA-002). Exactamente un mes después ocurrió la siguiente, contando con Jorge Glusberg, María Luisa Torrens, María Elena Ramos, Fábio Magalhães e Frederico Morais, además de Aracy Amaral (Fundo Aracy Abreu Amaral, Arquivo do IEB/USP, código de referencia AAA-BIELA-003).

I3 Fundo Aracy Abreu Amaral, Arquivo do IEB/USP, código de referencia AAA-BIELA-006 a 009.

I4 Fundo Aracy Abreu Amaral, Arquivo do IEB/USP, código de referencia AAA-BIELA-044 y 046.

I5 La relación completa de los invitados y asistentes a la reunión aparece descrita en el Relatorio final da Reunião de Consulta de Críticos da América Latina (Fundo Aracy Abreu Amaral, Arquivo do IEB/USP, código de referencia AAA-BIELA-046). Asimismo, en el mismo documento se recoge los motivos de ausencia de algunos de los críticos de arte como, por ejemplo, Marta Traba. Traba, aunque no asistió la reunión, envió una comunicación escrita que fue leída en el plenario de la reunión (Fundo Aracy Abreu Amaral, Arquivo do IEB/USP, código de referencia AAA-BIELA-043 y 046). 
arte latinoamericano contemporáneo. Cómo sabemos por el informe conservado en el fondo AAA y también por reflexiones académicas sobre el tema (MORETHY COUTO, 20I7; BARRAGÁN, 20I9), finalmente en la última sesión plenaria se optó por la continuidad de un evento de carácter internacional, no latinoamericanista.

Esta decisión truncó el seguimiento y la elaboración de una narrativa latinoamericanista respaldada por un evento oficial, consolidado y establecido, como era la Bienal de São Paulo. Este hecho ocasionó cierta frustración en Amaral, quien veía aquel acto como "una manifestación insostenible de desconfianza en su arte propio con valor autónomo, de preocupación por mantenerse atados al sistema del arte de los grandes centros mundiales" (AMARAL, I98I, p. 36).

Si bien es cierto, cómo apunta Morethy Couto (20I7), que pasarían dos décadas hasta que en Brasil se organizase otro certamen artístico con carácter integrador latinoamericanista - la Bienal del Mercosur en 1997-, este lapsus no eclipsó la defensa por la producción de un arte latinoamericano autónomo, no derivativo o exotizante, por algunos de los intelectuales del país - principalmente Aracy Amaral y Frederico Morais - relacionados con varios de los nombres recurrentes a lo largo de este texto.

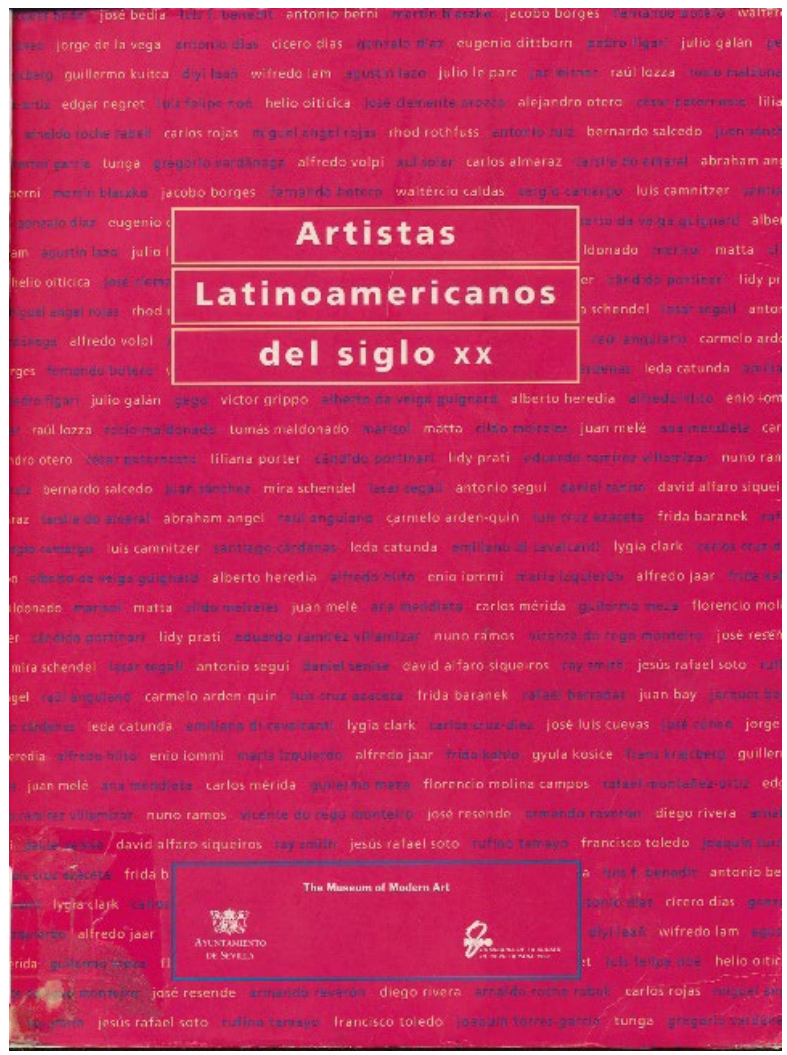

Figura 4-Cubierta del catálogo de la exhibición Artistas Latinoamericanos del siglo XX, celebrada en la Estación Plaza de Armas de Sevilla, durante la Exposición Universal de 1992 
Siguiendo las pistas dejadas por el fondo AAA, llegamos a la emblemática exposición Artistas Latinoamericanos del Siglo $\mathrm{XX}^{16}$ (Figura 4), una enciclopédica muestra con más de 400 obras, ideada por Waldo Rasmussen - director del Programa Internacional del Museum of Modern Arte (MoMA) - en el paradigmático año de I992, momento de las conmemoraciones del V Centenario de la llegada europea en América (RIBEIRO DOS SANTOS, 20I9). La curaduría de Rasmussen fue asesorada por Io especialistas latinoamericanos, incluyendo Aracy Amaral y otros profesionales, por veces citados en este escrito ${ }^{\mathrm{T}}$. En el fondo AAA relacionado con Artistas latinoamericanos... encontramos a I23 documento ${ }^{18}$, que pueden resultar esenciales para entender este nuevo impulso a la "idea del arte latinoamericano" ocurrido en aquellas fechas ${ }^{\mathrm{I9}} \mathrm{y}$ que incidió en distintos países europeos y en los Estados Unidos.

Entre tanto, cabe apuntar que el interés de Rasmussen - y del MoMA por asimilación - no fue algo novedoso. Como se recoge en el catálogo de la muestra, el comisario venía trabajando desde los años I960 con artistas y colecciones de América Latina, buscando su introducción en el mapa central del arte (RASMUSSEN, I992). Este tejido de relaciones con el MoMA y con Rasmussen, también podemos confirmar con el análisis del material del fondo del IEB/USP: algo más de una decena de documentos describen su actuación en el Latin American Committee organizado por el MoMA ${ }^{20}$, en el mismo año de aquella primera bienal latinoamericana, I978.

Con esta breve e inicial revisión sobre el archivo documental reunido por Aracy Amaral relacionado a sus labores "latinoamericanistas" queríamos dejar patente como, desde la investigación de este tipo de fuentes documentales, podemos trazar caminos y puentes entre los agentes que promovieron, teorizaron y forjaron determinado momento cultural. Este tipo de análisis nos conduce a investigaciones que apartan

I6 La exposición se exhibió en la Plaza de Armas de Sevilla, durante la celebración de la Exposición Universal de I992 entre el II de agosto y el I2 de octubre de I992. Posteriormente, fue instalada en el Musée National D’Art Moderne Centre Georges Pompidou y Hotêl des Arts (Io de noviembre de I992 a II de enero de I993), Joseph-Haubrich Kunsthalle (8 de febrero a 25 de abril de 1993) y finalmente en el MoMA (7 de junio al 7 de septiembre de I993).

I7 Los asesores de la exposición fueron: Aracy Amaral, Rita Eder, Paulo Herkenhoff, Ariel Jiménez, Ángel Kalenberg, Susana Torruella Leval, Lilian Llanes, Samuel Paz, Sylvia Pandolfi y Eduardo Serrano.

Fundo Aracy Abreu Amaral, Arquivo do IEB/USP, código de referencia AAA-LAATC-ooI a I23.

I9 A finales de los 80 e inicios de la década de I990 se revivió el interés por la difusión y promoción del arte latinoamericano con exposiciones, inauguración de instituciones, simposios, etc. Para ampliar sobre el tema, véase Ribeiro dos Santos, 20I9. Como anécdota que evidencia este incremento de la actividad, en una carta que Aracy Amaral envía a Rasmussen en 13/7/I992, se excusa de no poder asistir a la inauguración de Artistas Latinoamericanos... en Sevilla, pues en fechas posteriores, muy cercanas, tendría que viajar a las Islas Canarias para un simposio relacionado a la exposición Voces de Ultramar: Arte de América Latina y Caribe, I9IO - I960 (Fundo Aracy Abreu Amaral, Arquivo do IEB/USP, código de referencia AAA-LAATC -056). 
el foco del objeto, complejizando las redes relaciones e evidenciando el complejo enmarañado social, histórico y político que enmarca el hecho artístico y cultural.

\section{SOBRE A AUTORA}

RENATA RIBEIRO DOS SANTOS é doutora em História pela Universidade de Granada. Atualmente é professora e pesquisadora do Departamento de História da Arte e Musicologia da Universidade de Oviedo (UNIOVI), Espanha. E-mail: ribeirorenata@uniovi.es https://orcid.org/oooo-0002-2008-I579

\section{REFERÊNCIAS}

AMARAL, Aracy. Críticos de América Latina votan contra una bienal de Arte Latinoamericano. Revista del Arte y la Arquitectura en América Latina de Hoy, Medellín, v. 2, n. 6, I98I, p. 36-4I. . Marta Traba: décadas vulnerables. Arte en Colombia: Internacional, Bogotá, n. 23, I984, p. I7-I9. (Coord.). Arquitectura neocolonial: América Latina, Caribe, Estados Unidos. São Paulo: Memorial da América Latina y Fondo de Cultura Económica, I994.

. Damián Bayón: um olhar sobre a América. São Paulo: Fundação Memorial da América Latina, I995.

BARRAGÁN, Paco. I978: el año en que América Latina dejó de creer en sí misma. Artishock: Revista de Arte Contemporáneo, s.p., 20I9. Disponível em: <https://artishockrevista.com/20I9/04/I5/1978-el-ano-enque-america-latina-dejo-de-creer-en-si-misma >. Acesso em: Io fev. 2020.

BAYÓN, Damián (Coord.). América Latina en sus artes. Madrid: Siglo XXI Editores y Unesco, I974. (América Latina en su cultura).

FUNDAÇÃO BIENAL DE SÃO PAULO. Catálogo da I Bienal Latino-americana de São Paulo. Fundação Bienal de São Paulo, São Paulo, I978.

MORETHY COUTO, Maria de Fátima. La cuestión latinoamericana en las bienales realizadas no Brasil. Caiana, Buenos Aires, n. Io, 20I7, p. 48-60.

MOYSSÉN, Xavier. Artes plásticas na Semana de 22, de Aracy Amaral. Anales del Instituto de Investigaciones Estéticas, Ciudad de México, v. XII, n. 48, I978, p. I85-I96.

RASMUSSEN, Waldo (Coord.) Artistas latinoamericanos del siglo XX. Sevilla: Tabapress, 1992.

RIBEIRO DOS SANTOS, Renata. Arte Contemporáneo Latinoamericano en España: dos décadas de exposiciones (I992-20I2). Granada: Editorial UGR, 20I9. 